\title{
L'implementazione dei servizi in iGuzzini illuminazione
}

\author{
TONINO PENCARELLI ${ }^{*}$ ANDREA BURATTI ${ }^{* \star}$
}

\begin{abstract}
Obiettivo del paper: approfondire il fenomeno della servitization dal punto di vista delle attività di implementazione dei processi di service management.

Metodologia: caso studio singolo sviluppato su una grande azienda italiana del settore del lighting, realizzato seguendo rigorosamente le indicazioni della letteratura sul metodo dei casi.

Risultati: dall'analisi emergono l'importanza della cultura del servizio, di risorse umane dedicate, di strumenti multimediali, di continui processi di formazione interna ed esterna e, soprattutto, la criticità del coinvolgimento del prescrittore.

Limiti della ricerca: considera un solo caso e quindi non possono essere effettuate generalizzazioni.

Implicazioni pratiche: fornisce spunti di riflessione e idee operative d'ausilio ai manager delle imprese manifatturiere che intendono riqualificare la propria offerta di valore.

Originalità del lavoro: il contributo arricchisce la tradizionale prospettiva di analisi del "sistema di offerta incrementata" di Grönroos, mostrando come in un mercato complesso la creazione del valore necessiti del coinvolgimento di prescrittori (architetti, ingegneri, light designer) ed altri attori del canale (distributori, installatori).
\end{abstract}

Parole chiave: servitization; creazione del valore; offerta incrementata; mercato del lighting

Purpose of the paper: investigating the servitization phenomenon from the point of view of implementation activities.

Methodology: a single case study on large Italian enterprise in lighting market.

Findings: from the analysis the following main points emerge: the importance of service culture, the importance of human resources dedicated to evolve multimedia supports, the importance of the continuous processes of internal and external training and, above all, the critical involvement of the prescriber.

Research limitations: one case study is considered and it's impossible to make generalizations.

Ordinario di Economia e Gestione delle Imprese - Università degli studi di Urbino Carlo Bo

e-mail: tonino.pencarelli@uniurb.it

** Dottorando di Ricerca di Economia e Management - Università degli studi di Urbino Carlo Bo

e-mail: andrea.buratti@uniurb.it 
Practical implications: the paper provided reflections and operative ideas for the manager of the manufacture firms that want to requalify their value offer.

Originality of the study: it enriches the traditional perspective of the analysis of the "augmented service offering" by Grönroos, showing how in a complex market value creation requires the involvement of prescribers (architects, engineers, lighting designers) and of the other players in the channel (distributors, installers).

Key words: servitization; value creation; augmented service offering model; lighting market

\section{Introduzione}

Nel nuovo millennio e nell'era della "competizione di servizi” (Grönroos, 2009), anche per le imprese manifatturiere si afferma l'esigenza di affrontare le strategie competitive superando la tradizionale impostazione della Good Dominant Logic ed assumendo la prospettiva della Service-Dominant Logic, secondo cui i servizi sono centrali nell'offerta dell'impresa e diventano persino più importanti dei beni stessi (Vargo e Lusch, 2004). Una degli aspetti centrali di questa nuova prospettiva è l'integrazione di beni e servizi in un sistema di offerta ad alto contenuto immateriale e di servizi che nella letteratura internazionale è stata definita con termine servitization per identificare la trasformazione nel modello di business (Vandermerwe e Rada,1988).

Molti sono gli autori che hanno studiato il fenomeno evidenziando gli aspetti critici della trasformazione (Baines et al., 2009; Oliva e Kallenberg, 2003; Martinez et al., 2010) e le performance delle imprese "servitizzate" (Coyne, 1989; Gebauer e Friedli, 2005; Mathe e Shapiro, 1993; Mathieu, 2001; Neely, 2009; Oliva e Kallenberg, 2003; Wise e Baumgartner, 1999). Nonostante ciò, si rileva una scarsità di contributi sul come le imprese effettivamente implementano questo cambiamento.

Obiettivo del lavoro è approfondire il fenomeno della servitization dal punto di vista delle attività di implementazione dei processi di service management, illustrando come un'impresa manifatturiera attua e innova le attività di vendita e di consegna del valore al cliente mediante una strategia di servizio.

A questo scopo, è stato utilizzato il metodo del case study (Yin, 2009) in quanto permette di analizzare in profondità le variabili in gioco. Il caso è stato studiato avvalendosi del modello concettuale del sistema di offerta incrementato di valore di Grönroos (2009), che si è ritenuto particolarmente appropriato per illustrare ed interpretare il contenuto di servizi presente nell'offerta economica di imprese anche di tipo manifatturiero. Modello che, come si vedrà nel seguito, è stato arricchito ed integrato, inserendo nel sistema di servizi aziendali anche quelli attivabili mediante network di relazioni con la rete vendita ed il mondo dei professionisti e dei prescrittori.

Nel paragrafo due viene richiamata la letteratura di riferimento con particolare attenzione modello di Grönroos utilizzato nell'analisi del caso studio. Successivamente nel paragrafo tre viene illustrato il procedimento metodologico seguito e vengono riportati i dati raccolti in iGuzzini illuminazione, un'azienda 
manifatturiera operante nel settore del lighting nel paragrafo quattro. Infine, alla luce dei risultati ottenuti, si propongono riflessioni concettuali sul modello teorico di lettura del sistema di offerta, si indicano le principali implicazioni manageriali emerse dallo studio evidenziando i limiti della ricerca e suggerendo possibili futuri sviluppi di analisi nel paragrafo cinque.

\section{La creazione del valore nei processi di service management}

La Service-Dominant Logic si afferma come un orientamento culturale e strategico nel modo di fare business dell'impresa i cui principi di base possono essere sintetizzati in dieci punti chiave (Vargo e Lusch, 2007):

1. l'implementazione della conoscenza e delle competenze specialistiche deve essere considerata come la fondamentale unità di scambio;

2. gli scambi indiretti (beni, moneta, ecc.) mascherano la fondamentale unità di scambio;

3. i beni sono un supporto fisico ed un meccanismo di distribuzione per la fornitura di servizi;

4. la conoscenza è la fondamentale fonte di vantaggio competitivo;

5. tutte le economie sono economie di servizio;

6. il consumatore è sempre un co-produttore;

7. le imprese possono solo realizzare un'offerta di valore;

8. la service-dominant logic è customer oriented e relazionale;

9. le organizzazioni esistono per integrare e trasformare competenze specializzate di servizi complessi dispersi in un network;

10. il valore è sempre unico e determinato da parte del beneficiario.

Alla base di questa concezione vi è l'idea che la moderna situazione competitiva di gran parte dei mercati sia quella che Grönroos (2009) definisce "concorrenza di servizi", in quanto la soluzione di base dell'impresa (si tratti di un servizio o di un bene materiale) è semplicemente un prerequisito, ma non è condizione sufficiente per ottenere e mantenere un vantaggio competitivo. Le aziende di fatto sono in concorrenza fra di loro sulla capacità di offrire un prodotto (un'offerta economica integrata) dato dalla combinazione di beni e servizi (Kotler, 1967; Levitt, 1962; Rispoli e Tamma, 1992; Tunisini, 2002; Vandermerwe e Rada, 1989; Manzini e Vezolli, 2003; Paiola e Rullani, 2009) in grado di aumentare il valore complessivo dell'offerta aziendale. Ciò che conta è entrare in competizione con l'intero pacchetto, definito anche Product-Service System (Goedkoop et al., 1999), nel quale il singolo bene è soltanto un punto di partenza per lo sviluppo del vantaggio competitivo, non rappresentando da solo, una garanzia di successo. La competizione fra servizi non è sicuramente una novità, poiché le imprese come le banche, gli intermediari commerciali o le aziende di trasporto hanno da sempre operato in una simile situazione competitiva. Elemento distintivo è che questa logica coinvolge ed interessa ormai tutte le aziende e tutti i settori; pertanto, nel formulare ed implementare le strategie competitive, le imprese hanno l'imperativo di proporre al mercato offerte economiche ad alto contenuto di valore immateriale e simbolico, 
assumendo la filosofia di gestione tipica delle imprese dei servizi (Pencarelli, 2013).

Le imprese manifatturiere, quindi, si trovano dinanzi ad un cambiamento epocale nel modo di competere: esse devono avviare un cambiamento nei processi interni che viene definito "servitization" da Vandermerwe e Rada (1988), con cui le aziende integrano la tradizionale offerta di beni fisici con i servizi, allestendo un sistema di offerta di valore mediante un processo di innovazione organizzativa e dei processi (Baines et al., 2009). Altri autori considerano la servitization con un approccio evolutivo (Oliva e Kallenberg, 2003), mentre altri ancora (Martinez et al., 2010) hanno evidenziato come non tutte le imprese hanno lo stesso livello di servitization: a seconda del valore di base dell'attività svolta, della strategia di produzione, del ruolo degli asset e della tipologia di offerta, è possibile collocare le imprese lungo un continuum secondo un diverso grado di servitization. Altri autori, invece, focalizzano i loro studi sui benefici (Coyne, 1989; Gebauer e Friedli, 2005; Mathe e Shapiro, 1993; Mathieu, 2001; Neely, 2009; Oliva e Kallenberg, 2003; Wise e Baumgartner, 1999) dell'implementazione della servitization.

I vari contributi considerano la servitization un veicolo di creazione di valore per il cliente e per il rafforzamento della posizione competitiva, sicché i servizi dell'offerta devono essere progettati ed allestiti considerando analiticamente tutte le componenti, tenendo conto della prospettiva del mercato. Zeithaml (1988), in particolare, considera il valore come una valutazione globale del consumatore dell'utilità di un prodotto basata sulle percezioni di ciò che egli riceve (get) e ciò che egli dà (give), ben diverso dal rapporto qualità prezzo. Nei benefici (get) rientrano le componenti utilitaristiche, simboliche, edonistiche, estetiche ed emozionali, mentre nei sacrifici (give) vengono considerati gli sforzi ed il tempo speso per la raccolta delle informazioni, per il reperimento fisico del prodotto, per l'apprendimento del funzionamento, la manutenzione ed i costi psicologici del rischio (Menon et al., 2005; Ulaga e Chacour, 2001). Nella definizione degli elementi che costituiscono l'offerta di valore dell'impresa devono essere inoltre effettuate due ulteriori considerazioni.

In primis va tenuto conto del fatto che il valore si crea continuamente, in un rapporto di lungo periodo tra fornitore e cliente; in effetti, la creazione del valore non avviene solo nell'ambito di una singola transazione isolata tra fornitore e cliente, ma nell'ambito di un orizzonte temporale di lungo termine, dove la singola transazione rappresenta solamente un episodio, sicché, sono la relazione e la bontà di questa a creare valore. In termini analitici il valore dipende dal rapporto fra $i$ benefici ed i sacrifici che si sviluppano lungo tutta la relazione con il cliente (Grönroos, 2009).

Inoltre, è importante tenere in considerazione il ruolo che può giocare il cliente "nel processo di value creation"; esso non è solo un soggetto passivo, ma un soggetto che può partecipare attivamente sul piano fisico, intellettuale ed emotivo alla creazione del valore ed è proprio il suo coinvolgimento che accresce il valore dell'offerta, incrementandone i benefici netti per esso e per l'impresa. La partecipazione attiva del cliente nei processi di creazione del valore consente infatti all'azienda di conseguire una serie di vantaggi, come la possibilità di ottenere 
margini più elevati, un miglioramento della fedeltà del cliente ed il rafforzamento del potenziale innovativo (Prahalad e Ramaswamy, 2000). Di conseguenza, le risorse dedicate a sviluppare la relazione con il cliente vanno considerate come importanti investimenti di lungo termine, piuttosto che come costi. Nell'implementare i processi di service management, le imprese devono essere tuttavia consapevoli che non tutti i clienti saranno disposti o vorranno partecipare allo stesso modo al processo di creazione del valore, e che il valore attribuito all'offerta non sarà uguale per tutti e in tutte le occasioni. Ciononostante, emerge come la sfida per le imprese che operano negli attuali contesti di competizione di servizi sia non tanto del se, ma di come integrare concretamente i processi di servizio alla loro tradizionale offerta. In letteratura esistono vari contributi sulle variabili chiave del processo di trasformazione e di servitization (Martinez et al., 2010; Ahamed et al., 2013), ma come sottolineato anche da Baines et al., (2009), c'è una scarsità di contributi che analizzano come le imprese manifatturiere hanno integrato nei loro processi di vendita questa nuova logica. Per analizzare questo cambiamento è stato preso in considerazione il modello dell'offerta incrementata di Grönroos (2009).

\subsection{Un modello per la definizione della product offering}

Consolidato che il servizio rappresenta una componente fondamentale per la creazione di valore anche per le imprese manifatturiere, è necessario soffermarsi sul come questi elementi possano confluire nella progettazione dell'offerta. Grönroos (2009) definisce il modello dell'offerta incrementata per guidare il management nella progettazione dell'offerta di servizi finalizzata all'accrescimento di valore per la domanda e per l'impresa. Anche se tale modello è stato sviluppato nel contesto del service management, riteniamo che esso possa essere un valido strumento metodologico applicabile in tutti i contesti poiché, come più volte ribadito, la competizione di servizi rappresenta il nuovo paradigma concorrenziale. La capacità del modello di adattarsi a contesti differenti da quello originario risiede nel fatto che esso non si focalizza sul "che cosa" deve essere fornito al cliente, ma soprattutto sul "modo" con cui il valore deve essere fornito e comunicato. Attraverso questo modello possono essere mappati e considerati operativamente tutti gli elementi riconducibili ai benefici ed ai sacrifici della formula del valore, compresa la partecipazione del cliente. Infine, il modello si adatta anche all'analisi di rapporto di medio-lungo termine tra fornitore e cliente, in quanto gli elementi considerati non escludono la possibilità di interazioni ripetute fra l'impresa ed il cliente.

Il modello di Grönroos (2009) si basa su quattro aspetti essenziali :

1. il concetto di servizio;

2. il pacchetto di servizi, ovvero il che cosa si offre al target;

3. i processi connessi al sistema di erogazione dei servizi, cioè la specificazione del come, dove, quando, chi, ruolo cliente, qualità-produttività;

4. i processi di comunicazione, sia interni che esterni. 
Fig. 1: Modello dell'offerta incrementata

IMPRESA PRODUTTRICE

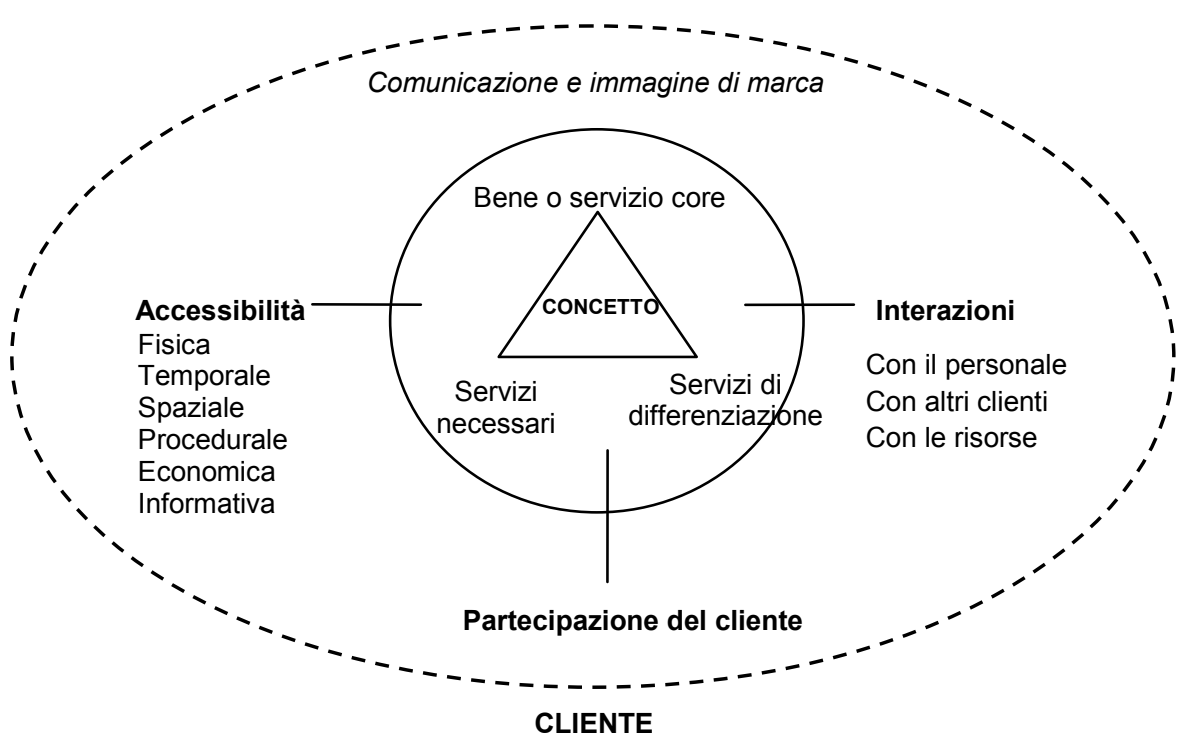

Fonte: Adattamento da Grönroos, 2009

Il concetto di servizio qualifica il bisogno e le funzioni che il servizio è finalizzato a soddisfare ai fini dell'implementazione della logica "della value cocreation". Questo aspetto deriva dallo studio della domanda ed è essenziale ai fini della progettazione del valore nell'ambito dei confini del business in cui si intende operare, imponendo la presenza nelle imprese di una radicata cultura del servizio che consente ad esse di comprendere sia le esigenze della domanda e la prospettiva del valore della clientela, sia le modalità per attuare efficaci processi di erogazione della prestazioni.

Il nucleo centrale del modello considera l'offerta dell'impresa come un pacchetto composto dall'elemento essenziale dell'offerta, sia esso un bene o un servizio e dai servizi ausiliari (necessari) e di facilitazione (di differenziazione). I beni e servizi "core" qualificano e sono strettamente connessi alla mission dell'impresa, mentre i servizi ausiliari e di facilitazione sono attività intangibili che arricchiscono e differenziano l'offerta. Tuttavia lo stesso Grönroos (2009) riconosce che questa suddivisione ha dei confini sfumati in quanto, a seconda dei contesti, uno stesso servizio può essere qualificato come necessario oppure di differenziazione. Secondo Ordanini (1999), nel caso di servizi ad alto valore aggiunto alle imprese, come ad esempio la consulenza strategica, tutti i servizi devono essere progettati in modo da agire sulle risorse alla base del vantaggio competitivo del cliente. La sostituzione del servizio essenziale con un bene non modifica la struttura concettuale del modello, per cui è possibile sostenere la sua validità anche per imprese non classificate di servizi nelle statistiche ufficiali. 
Per creare valore al cliente occorre poi definire le modalità con le quali il pacchetto di offerta entra nelle disponibilità del target, individuando "il come" aumentare i benefici e ridurre i sacrifici mediante l'allestimento dell'insieme dei processi (attività) connessi con il sistema di erogazione dei servizi. Sistema che va opportunamente schematizzato, mappando le varie fasi ed i vari momenti di contatto tra domanda ed offerta (momenti della verità o service encounters, che avvengono tra clienti e front office aziendale) o tra servizi interni (back office) e servizi esterni (front office).

Per ogni pacchetto di base l'impresa dovrà studiare anche il modo con cui $\mathrm{i}$ servizi vengono erogati e quindi definire l'accessibilità, l'interazione e la partecipazione del cliente. L'accessibilità del servizio è la facilità con cui l'impresa permette agli utenti di fruire del servizio e questa può essere declinata in accessibilità (Pencarelli, 2013):

- fisica, ovvero la facilità per gli utenti a entrare in contatto con le risorse fisiche dell'impresa compreso il personale di front-office;

- temporale, ossia gli orari di accesso al servizio;

- spaziale, cioè la facilità di raggiungimento del punto di erogazione del servizio;

- procedurale, ovvero la semplicità con cui gli acquirenti possono attivare le procedure;

- economica, fa riferimento al prezzo e a tutti i costi monetari per usufruire di un determinato servizio;

- informativa, che attiene alla qualità ed alla fruibilità delle informazioni per i clienti.

L'interazione riguarda tutte le relazioni che si vengono a creare fra il cliente ed i sistemi di erogazione dell'impresa. Le interazioni ben sviluppate facilitano la fruizione dell'offerta aziendale ed accrescono il valore percepito dal cliente. Possono essere considerati in questo livello le interazioni:

- con il personale di front-office, gli agenti, i tecnici e più in generale con i dipendenti dell'impresa;

- con risorse fisiche del sistema di produzione/erogazione del servizio;

- con i sistemi che permettono la realizzazione del servizio;

- con gli altri clienti che simultaneamente sono coinvolti nel processo di erogazione del servizio.

La partecipazione del cliente è il contributo che esso può fornire alla progettazione, all'erogazione, al controllo, alla commercializzazione, alla comunicazione (passaparola) del servizio: come evidenziato precedentemente, il coinvolgimento del cliente aumenta notevolmente il valore percepito dalla domanda, agendo sia dal lato dei benefici (psicologici, sociali, simbolici, economici, di apprendimento), che dei sacrifici (economici, di tempo, di relazione, ecc.).

La comunicazione, infine, riguarda sia quella spontanea, derivante dalle esperienze dei clienti e dal passaparola da essi alimentato, sia quella pianificata dall'impresa. Essa gioca un ruolo cruciale e crescente nell'ambito dei mercati ove le offerte economiche sono ad elevato contenuto d'immaterialità e simbolico e dove pertanto il comunicare ha l'importante e difficile compito di far comprendere al 
pubblico le basi del valore dell'offerta. La comunicazione, inoltre, può accrescere o diminuire il valore della marca dell'impresa o dell'offerta e pertanto impone alle aziende accorte politiche di comunicazione e un monitoraggio continuo delle opinioni del pubblico. Infatti, nei contesti ove i prodotti sono complessi ed altamente immateriali è difficile spiegare le fonti del valore dell'offerta e quindi ai fini della reputazione l'importanza del passaparola si accresce, imponendo alle imprese (rispetto alle aziende orientate da una "good dominant logic") una gestione più attiva e consapevole di questi fenomeni, siano essi espressi nelle reti sociali tradizionali che nei social network on line.

$\mathrm{Va}$ inoltre posta estrema attenzione alla comunicazione face to face con il cliente, in occasione dei "momenti della verità". Per questo è importante accrescere la cultura del servizio e le competenze relazionali del personale di contatto, in modo da migliorare l'empatia con la clientela, le abilità di problem solving o di recupero di disservizi e l'attitudine a considerare il valore frutto della cooperazione tra fornitori e clienti, sia esterni che interni. Per questo, tali processi non possono limitarsi a coinvolgere solo gli esperti di marketing o coloro che sono a stretto contatto con la clientela, ma vanno implementati in modo diffuso all'interno e fuori dei confini organizzativi, nell'ambito del sistema del valore. Nelle aziende, sia i full-time marketers, ovvero gli specialisti della funzione di marketing o delle vendite, sia i part-time marketers, ovvero specialisti in aree aziendali lontane dal marketing che tuttavia hanno interazioni con la clientela, devono avere una cultura di marketing e del servizio, perché anche da essi dipenderà la soddisfazione del cliente. Il personale si configura, quindi, come un fattore chiave nel cambiamento e necessita di essere opportunamente considerato e valorizzato. Agli occhi del cliente sono proprio le competenze e le capacità relazionali del personale che si manifestano nei momenti della verità, ad influenzare la soddisfazione del cliente e determinare un elemento di differenziazione rilevante rispetto ai concorrenti. Da non sottovalutare l'importanza della partecipazione ai blog o la frequentazione dei social network per favorire reclami o suggerimenti dei clienti (numeri verdi, cassette delle idee, ecc.). Sarà cruciale anche attivare azioni di comunicazione e formazione interna, con l'obiettivo di creare, mantenere e potenziare $i$ rapporti interni tra $i$ dipendenti dell'organizzazione e rafforzare le competenze e le capacità necessarie per fornire performance efficaci.

\section{Metodologia dell'indagine}

In questa parte dello studio, si vuole illustrare ed approfondire come un'impresa manifatturiera evolva verso il paradigma del servitization ed implementi processi di service management nell'ambito delle strategie competitive. A questo scopo si ricorre al metodo del case study, qui considerato come metodo di ricerca preferibile e funzionali ai nostri obiettivi in quanto permette di analizzare in profondità le variabili in gioco rispetto ad altre metodologie (Fattore, 2005; Corbetta, 1999). 
Un campionamento teorico (Patton, 2002) ha permesso di individuare la iGuzzini illuminazione come caso rappresentativo del fenomeno oggetto di ricerca. L'azienda, che può essere classificata secondo i parametri Eurostat come una grande impresa in quanto ha conseguito nel 2011 un fatturato pari a 187 Milioni di Euro, capitale sociale 20 Milioni di Euro e un numero di dipendenti di 1.117 unità, ha sede a Recanati, in Provincia di Macerata e produce apparecchi per l'illuminazione posizionati nella fascia alta del segmento tecnico. La iGuzzini illuminazione, che si rivolge al segmento premium del mercato, è stata scelta come oggetto di indagine perché secondo la classificazione ATECO 2007 rientra fra le attività manifatturiere (Codice 27.40.09) e, secondo alcune interviste ad esperti del settore, aveva già implementato i servizi nella propria offerta. Inoltre, le caratteristiche del settore degli apparecchi per l'illuminazione identificato con il termine "lighting (Lojacono, 2005), si presta meglio di altri settori per analizzare l'integrazione fra beni e servizi per molteplici motivi:

- il vitale ruolo giocato dalla conoscenza nella realizzazione dei prodotti;

- la competizione su scala globale;

- l'importanza dell'innovazione tecnologica nel settore;

Tutti questi elementi, unitamente al fatto che il settore del lighting è poco considerato dalla letteratura manageriale, fanno della iGuzzini illuminazione una realtà che si presta ad essere considerata come un caso rappresentativo del fenomeno oggetto di indagine.

Per la realizzazione dello studio sono state utilizzate tre fonti informative, che hanno permesso una triangolazione dei dati (Jick, 1979) al fine garantire la validità dei risultati. Nello specifico:

1. interviste in profondità semistrutturate;

2. questionario strutturato;

3. fonti secondarie (presentazione aziendale, sito internet ed altri documenti aziendali).

I quattro punti del modello di Grönroos e le relative considerazioni sul valore hanno costituito la traccia delle interviste in profondità.

La raccolta dei dati, avvenuta fra il dicembre del 2012 a marzo 2013, si è articolata nelle seguenti fasi (Lamberti e Noci, 2010):

- è stato contattato 1'Ufficio del Personale iGuzzini illuminazione spiegando brevemente le finalità della ricerca, gli obiettivi, le fasi, e gli strumenti di raccolta;

- dopo aver accordato la collaborazione, la Responsabile del Personale ha selezionato 5 manager di prima linea in diverse posizioni dell'azienda che hanno gentilmente accettato di essere intervistati;

- sono state realizzate e registrate 5 interviste ai 5 manager selezionati con una durata media di circa 40 minuti (Tab.1);

- le interviste sono state trascritte ed il testo è stato analizzato con l'ausilio del software NVIVO 7. Questo ha permesso di organizzare il verbatim delle interviste e analizzare efficacemente il testo in funzione degli obiettivi dell'indagine; 
- sulla base dei risultati ottenuti dall'analisi testuale, è stato realizzato un questionario strutturato somministrato in modalità CAWI (Computer Assisted Web Interviewing) a 15 persone scelte casualmente in azienda, diverse dai 5 intervistati, per triangolare le evidenze emerse dalle interviste in profondità. Lo scopo del questionario non era misurare, ma effettuare una valutazione da parte di altri soggetti delle dichiarazioni fornite dagli intervistati;

- i risultati sono stati corroborati da fonti secondarie come la presentazione ufficiale aziendale, il sito web ed altri documenti messi a disposizione dall'azienda;

- al fine di verificare la validità dei risultati ottenuti, una sintesi del lavoro è stata sottoposta e discussa con i manager iGuzzini illuminazione.

Tab. 1: Caratteristiche intervistati ed interviste

\begin{tabular}{|c|c|c|c|c|c|}
\hline Id & Nome ufficio & Descrizione sintetica & Data & $\begin{array}{l}\text { Anni in } \\
\text { azienda }\end{array}$ & $\begin{array}{l}\text { Minuti } \\
\text { intervista }\end{array}$ \\
\hline $\mathrm{A} 1$ & $\begin{array}{l}\text { Research center \& } \\
\text { Communication }\end{array}$ & $\begin{array}{l}\text { Responsabile } \\
\text { comunicazione al } \\
\text { mercato }\end{array}$ & $13 / 12 / 12$ & 32 & 40 \\
\hline $\mathrm{A} 2$ & Lighting engineering & $\begin{array}{l}\text { Progettazione } \\
\text { illuminotecnica estero }\end{array}$ & $13 / 12 / 12$ & 25 & 43 \\
\hline A3 & After sales & $\begin{array}{l}\text { Responsabile assistenza } \\
\text { post-vendita }\end{array}$ & $13 / 12 / 12$ & 7 & '42 \\
\hline A4 & $\begin{array}{l}\text { Competitive } \\
\text { Intelligence }\end{array}$ & $\begin{array}{l}\text { Responsabile indagini ed } \\
\text { analisi della concorrenza }\end{array}$ & $14 / 01 / 13$ & 32 & '32 \\
\hline A5 & Lighting Training & $\begin{array}{l}\text { Responsabile } \\
\text { formazione interna e al } \\
\text { mercato }\end{array}$ & $11 / 02 / 13$ & 12 & ‘54 \\
\hline
\end{tabular}

Fonte: Nostra elaborazione

\section{Risultati dell'indagine}

Coerentemente con altri case study presenti nella letteratura internazionale (Eisenhardt, 1989; Cook et al., 2006), si riporta di seguito quanto emerso dalle interviste, rinviando alle conclusioni (5) la loro interpretazione. I risultati vengono riportati in base al modello di Grönroos ed alle altre considerazioni emerse dalla letteratura. Nello specifico, viene approfondita innanzitutto la consapevolezza dell'importanza del servizio all'interno dell'azienda (4.1) e con quali servizi la iGuzzini illuminazione arricchisce i beni (4.2). Successivamente vengono analizzate le modalità di erogazione (4.3) ed approfondito il modo in cui il prescrittore concretamente aggiunge valore dell'offerta (4.4). Infine, s'analizzano le modalità con cui l'azienda comunica e fa formazione interna (4.5). 


\subsection{La consapevolezza dellimportanza del servizio}

In relazione all'importanza del servizio, il management iGuzzini illuminazioni afferma: "non puoi prescindere dal servizio perché è forse la leva di vendita più forte che hai" (A3). Inoltre: "la iGuzzini è un'impresa manifatturiera, progettando $i$ prodotti e realizzandoli in azienda, ma anche di servizi perché è un modo di vendere $i$ nostri prodotti che abbiamo sempre utilizzato che consiste nel dare servizio ai clienti fornendogli tutte le indicazioni per usare al meglio i nostri prodotti” (A2). Il servizio non è importante solo per la iGuzzini illuminazione, ma anche per le altre aziende del settore in quanto viene riconosciuto che nell'erogazione dell'offerta: "la differenza si colloca nella qualità del servizio. E quindi, più siamo incisivi, più siamo attenti, più siamo vicini proprio al servizio su misura e più il cliente percepirà la professionalità della iGuzzini." (A5).

L'importanza del servizio emerge ampiamente dai risultati del questionario: tutti i rispondenti infatti hanno sottolineato che il servizio è un elemento fondamentale per competere nel settore del lighting e non un semplice elemento differenziante o marginale dell'offerta.

Il ruolo cruciale del servizio viene messo in forte evidenza anche nell'ambito della mission dell'azienda, che viene così formalizzata nel sito aziendale (www.iguzzini.it): "La nostra missione è migliorare la qualità della luce e quindi la qualità della vita delle persone producendo apparecchi di illuminazione al massimo livello di qualità tecnica" per cui emerge che l'obiettivo dell'azienda non è la mera produzione di apparecchi di illuminazione, ma fornire progetti illuminotecnici capaci di esaltare la qualità della luce.

\subsection{L'arricchimento del bene fisico}

Dalle interviste emerge una chiara descrizione del business della iGuzzini illuminazioni: "Noi siamo un'azienda BtoB, ovvero non facciamo vendita di prodotti all'utilizzatore finale. I nostri prodotti vengono venduti attraverso una catena abbastanza articolata ed anche abbastanza lunga" (A1). A valle della catena la situazione è estremamente eterogena: in genere gli acquirenti o meglio $i$ committenti di un progetto sono costruttori edili, aziende private, oppure le istituzioni pubbliche che spesso non sono i veri utilizzatori del prodotto. Fra l'impresa produttrice e l'acquirente c'è uno strato intermedio costituito da numerose figure professionali le quali hanno il compito di prescrivere il prodotto d'illuminazione come architetti, light designer, progettisti d'illuminazione, progettisti d'impianto. Non meno importanti sono gli installatori i quali: "andranno ad installare fisicamente il prodotto nel cantiere" (A5). Da non sottovalutare infine il ruolo del distributore ovvero: "colui che vende il nostro prodotto, il cliente di fattura" (A5). Tralasciando la vendita dei prodotti da banco basata essenzialmente sul prezzo e sulla gestione della scontistica, la vendita su commessa è il vero segmento di riferimento della iGuzzini illuminazione poiché garantisce maggiori margini e quantitativi. Competere in questo segmento stravolge il ruolo dei vari attori del canale al punto 
che il vero cliente dell'impresa è il prescrittore e obbliga a qualificare l'offerta attraverso una serie di servizi ad esso destinati. Dalla documentazione aziendale emerge che la iGuzzini illuminazione offre modularmente e gratuitamente, i seguenti servizi:

- formazione, si svolge in una fase embrionale della relazione con il cliente business, quando può ancora non esserci un progetto, ed ha come obiettivo di spiegare l'importanza della progettazione illuminotecnica, le caratteristiche della progettazione in determinati contesti o nuove tecnologie;

- assistenza tecnica, l'azienda fornisce una serie di informazioni per favorire la prescrizione del prodotto in un determinato ambito applicativo;

- progettazione illuminotecnica, ovvero la realizzazione di un impianto di illuminazione in base ad un calcolo tecnico-scientifico. Nella progettazione illuminotecnica si ricorre sempre più all'utilizzo di nuovi strumenti multimediali (Dialux, Photoshop, 3DMax Studio, ecc.) per presentare efficacemente gli effetti che una soluzione illuminotecnica produce in un determinato ambiente;

- prodotti speciali, ovvero la personalizzazione del prodotto in base alle specifiche esigenze del cliente. Questa può andare da un livello soft, se vengono richieste delle semplici modifiche ad esempio al colore dei prodotti, oppure hard quando viene richiesto un prodotto nuovo;

- post-vendita, tutte le attività che supportano il cliente nel caso in cui si manifesti una difettosità del prodotto;

- assistenza in loco, consiste nel sopralluogo in cantiere che può essere realizzato sia nelle fasi pre-vendita che post-vendita, per acquisire le informazioni necessarie alla definizione del progetto oppure supportare i tecnici nella corretta installazione dei prodotti.

Il servizio principale dell'offerta iGuzzini è la progettazione illuminotecnica mentre gli altri servizi svolgono un ruolo di facilitazione. Questo servizio viene erogato nella fase pre-acquisto ed è fondamentale per trainare la vendita degli apparecchi di illuminazione ed erigere una barriera nei confronti della concorrenza. Le interviste hanno confermato l'effettiva erogazione dei servizi ed hanno permesso di stabilire che i servizi sono: "un vero e proprio arricchimento necessario" (A5). Gli intervistati sottolineano inoltre, che: "il servizio aggiunge valore, in quanto per un prodotto altamente tecnico più sale il livello tecnologico, più sale l'expertise ed il know-how dell 'azienda più c'è necessità di servizio" (A4).

I veri destinatari di questi servizi non sono però gli utilizzatori o altre figure, bensì i professionisti, siano essi light designer, architetti o ingegneri, che prescrivono i prodotti e stimolano in modo decisivo le vendite del distributore. Gli intervistati sostengono che: " $i$ servizi sono concentrati al professionista per facilitare la cosiddetta prescrizione degli apparecchi, cioè si offrono supporti affinché il progettista scelga un nostro apparecchio e lo metta all'interno del progetto illuminotecnico offerto al potenziale acquirente" (A1).

Emerge pertanto come la figura chiave per la iGuzzini illuminazione e forse per tutte le imprese del lighting, sia rappresentata dal prescrittore, poiché la maggior parte delle vendite viene realizzata su prescrizione, ossia su suggerimento di 
professionisti capaci di interpretare le esigenze degli utilizzatori e di tradurle nel linguaggio tecnico della produzione. Il prescrittore è quindi un attore chiave, una terza parte che tuttavia nelle fasi iniziali del processo di acquisto può essere considerato il vero cliente dell'azienda, anche se con esso non avvengono scambi monetari, perché permette di accedere ai progetti e di vendere i prodotti.

Come suggerisce la metodologia dello studio dei casi (Yin, 2009), si è fatto ricorso alla triangolazione, da cui è emerso che 13 rispondenti su 15 affermano che l'offerta è basata sull'apparecchio fisico opportunamente arricchita con una serie di servizi. Solo due rispondenti su 15 invece considerano che l'offerta iGuzzini illuminazione è costruita per competere sul prezzo.

\subsection{Partecipazione, interazione e accessibilità del cliente. Il ruolo dei prescrittori}

Il caso suggerisce che anche per un'impresa manifatturiera sia molto importante comprendere come il cliente, in questo caso mediato dal prescrittore (4.2), fruisca dei servizi considerando che non vale sempre il principio della simultaneità nell'erogazione e produzione del servizio. Dalle interviste emerge che l'accessibilità ai servizi avviene con diverse modalità e si possono verificare situazioni assai difformi a seconda che il progetto sia stato acquisito tramite un distributore, oppure un architetto. Inoltre, particolarmente rilevante è l'accessibilità informativa: per migliorarla la iGuzzini illuminazione ha investito molte risorse nel catalogo elettronico in modo che: "in maniera abbastanza autonoma il prescrittore può accedere alle risorse aziendali ciò̀ fogli d'istruzioni, schede tecniche, curve fotometriche, foto realizzazioni." (A3). Il prescrittore, talvolta anticipando i desideri e le esigenze dei clienti, partecipa al servizio in vari modi: "per indicare quali sono gli obiettivi che il progetto deve svolgere, poi tutta una serie di verifiche nello sviluppo del progetto per poter mettere appunto la soluzione. Possiamo chiamarlo una sorta di ping pong continuo. Anche nello sviluppo ad esempio di prodotti che vanno fuori gamma che noi chiamiamo prodotti speciali" (A1). Elemento centrale dell'interazione con il prescrittore (cliente) è l'agente di vendita. Infatti: "l'agente è colui che ha il contatto con il cliente e non lo abbandona mai. Anche quando viene in azienda per visitare, parlare con il direttore delle progettazione, comunque è sempre presente il commerciale di riferimento" (A5). Nella definizione degli aspetti operativi del progetto anche i tecnici possono avere relazioni con i prescrittori o con i collaboratori dei prescrittori, ma l'agente rimane l'interlocutore di riferimento, il responsabile della relazione e del budget del progetto. $\mathrm{E}$ come un vigile che accompagna il prescrittore lungo tutta la relazione con l'azienda e s'interfaccia con la struttura incaricata della progettazione illuminotecnica all'interno dell'azienda. Infine data l'eterogeneità delle situazioni, la partecipazione del cliente e l'accessibilità non si prestano ad una rigida pianificazione formale bensì ad una pianificazione informale basata su una serie di prassi, consuetudini e routine aziendali consolidatesi nel tempo. 
I risultati dell'analisi empirica confermano l'importanza attribuita dall'impresa alla partecipazione, all'accessibilità e all'interazione con il prescrittore, prima ancora che con il distributore o con il cliente finale. Risulta inoltre che il prescrittore viene considerato come un soggetto attivo che si informa e partecipa attivamente alla definizione del progetto mentre una leggera divergenza esiste nella valutazione delle dimensioni più importanti dell'accessibilità e nella responsabilità dell'interazione. Infatti, 10 su 15 rispondono che la dimensione più importante per la valutazione dell'accessibilità ̀̀ "la qualità e la facilità di fruizione delle informazioni" mentre 5 su 15 rispondono "la facilità per gli utenti d'entrare in contatto con il personale dell'azienda". Nella responsabilità dell'interazione, 11 su 15 sottolineano che il responsabile è l'agente commerciale mentre 4 su 15 rispondono che c'è più di un interlocutore senza un responsabile preciso.

\subsection{Come il prescrittore partecipa all'erogazione del servizio ed aggiunge valore all'offerta}

Dalle interviste emerge inoltre che la partecipazione del prescrittore, in quanto medium cognitivo ed informativo tra cliente e fornitore è di vitale importanza non solo per accedere ad una commessa ma anche per la definizione dell'offerta dato che il prescrittore: "porta valore perché è portatore esperienziale di una tipologia d'esigenza". Inoltre, il rapporto con il prescrittore permette di "indirizzare meglio gli sforzi dell'azienda per migliorare l' offerta e soprattutto comprendere gli sviluppi futuri del settore" (A4). L'interdipendenza del mercato del lighting con il settore edile, impatta sulla durata del rapporto fra azienda e prescrittore in quanto: "dal concepimento del progetto, all'avvio del progetto, all'esecuzione ed alla chiusura del progetto possono passare e passano spesso anni ed anni. Per cui il cliente va fidelizzato, il cliente può cambiare idea, non solo le condizioni possono cambiare, il progetto stesso può cambiare e cambia in corso d'opera" (A4). Si instaura quindi un naturale rapporto di lungo periodo fra iGuzzini ed il prescrittore che può addirittura scavalcare il singolo progetto ed arrivare ad una costante collaborazione su tutti i progetti acquisiti dal prescrittore. Si afferma infatti che "la relazione è importante e si costruisce sul servizio" (A5).

Queste evidenze sono state confermate dal questionario strutturato, in quanto i 15 rispondenti sono concordi nel sostenere l'importanza della partecipazione del prescrittore nel progetto e che la relazione con il prescrittore debba essere gestita in una logica di lungo periodo, essendo il prescrittore medium indispensabile per decodificare i linguaggi vari e indefiniti della domanda con quelli tecnici e complessi dell'offerta.

Infine, dall'archivio aziendale è stato trovato un messaggio pubblicitario a mezzo stampa della campagna "Partner for better light" del 2004- 2008 che conferma ulteriormente le evidenze in quanto, iGuzzini illuminazione esprimeva chiaramente la volontà di essere un partner nella realizzazione di un progetto edile e di collaborare con tutte le figure professionali della filiera 


\subsection{Comunicazione al mercato e formazione interna}

$\mathrm{Nel}$ settore del lighting la comunicazione è prevalentemente di tipo logicorazionale in quanto è rivolta generalmente ai professionisti, non ha un obiettivo comportamentale (acquisto, riacquisto, ecc.) bensì di sviluppare una conoscenza approfondita dei prodotti (Pastore e Vernuccio, 2006). Nel settore, il confine fra comunicazione e formazione è molto labile. Negli anni ottanta, parallelamente ai tradizionali strumenti di comunicazione (cataloghi, merchandising, ecc.), in iGuzzini illuminazione sono state realizzate campagne di comunicazione mass-market non di prodotto ma di corporate brand a mezzo stampa sui principali quotidiani italiani, per far conoscere l'azienda e sviluppare un atteggiamento positivo nell'opinione pubblica verso un'azienda che promuoveva la cultura della luce. Negli anni duemila le risorse si sono concentrate sulla comunicazione al prescrittore e la formazione del personale è diventata una variabile critica, avendo compreso che il personale rappresenta uno dei principali veicoli di comunicazione aziendale specie per aziende che offrono prodotti complessi con molti contenuti di conoscenza e di immaterialità. Il cambio di strategia ha permesso di creare maggiori occasioni di vendita, migliorare il posizionamento dell'azienda, consolidare le relazioni con i prescrittori ma soprattutto di sviluppare una crescente sensibilità alla progettazione illuminotecnica da parte del professionista e, di fatto, valorizzare il servizio fornito dall'azienda. In altre parole, il personale è l'elemento centrale nella comunicazione del valore del prodotto iGuzzini illuminazione e permette di giustificare il maggior prezzo. Come emerso dall'intervista: "è stato importante negli anni fare formazione al mercato per spiegare proprio il perché il prodotto deve avere quelle determinate caratteristiche. Il perché il prodotto veramente costa tanto" (A5).

La rilevanza della formazione è dimostrata dalle numerose attività sia interne che esterne. Dalle interviste emerge che la formazione interna è rivolta: "a tutte le persone che chiaramente hanno un ruolo tecnico o commerciale all'interno dell'azienda; "in modalità mista quindi on-line ed in aula e addirittura, cercando di essere sempre più innovativi sviluppando corsi e strumenti che possono essere fruibili nei tempi morti quindi attraverso anche Ipad ed altro" (A5). La formazione esterna invece riguarda il distributore e la sua forza vendita, ingegneri, architetti, light designer ed in particolare quelli situati in nuovi mercati come l'India, il Medio Oriente, la Cina e il Brasile. L'importanza della formazione viene corroborata dalle risposte del questionario strutturato in quanto, dai 15 rispondenti, emerge un'assoluta condivisione sull'importanza della formazione per un'azienda che si posiziona nella fascia alta del mercato del ligthing. Emerge inoltre che per 7 rispondenti su 15 la principale difficoltà nel comunicare il servizio è nella formazione del professionista, per 5 su 7 è semplice nei mercati tradizionali mentre per 3 su 15 è complesso comunicare il servizio a causa della natura intangibile.

Un ulteriore riscontro dell'importanza attribuita dalla iGuzzini illuminazione alla formazione si ritrova nell'organigramma aziendale: è presente infatti un'unità organizzativa specifica che si occupa di gestire e coordinare la formazione su scala globale. 


\section{Discussione dei risultati}

L'obiettivo del lavoro era approfondire il fenomeno della servitization dal punto di vista delle attività di implementazione dei processi di service management, illustrando come un'impresa manifatturiera attua e innova le attività di vendita e di consegna del valore al cliente mediante una strategia di servizio.

Innanzitutto, dai risultati dell'indagine, emerge che il mercato del lighting presenta una struttura complessa ed articolata, ben diversa quindi dai mercati consumer, composta da numerosi soggetti che a vario titolo contribuiscono alla creazione del valore. L'acquirente di beni problematici come sono gli apparecchi d'illuminazione, a causa della mancanza di informazioni e di asimmetrie di competenze rispetto all'offerta, partecipa limitatamente al processo di co-creazione di valore delegando la partecipazione ai prescrittori (ingegneri, architetti, light designer), che prescrivono i prodotti nella vendita su commessa, diventando $\mathrm{i}$ soggetti chiave nella creazione del valore e fungendo da mediatori di codici comunicativi tra il mondo dei consumi ed il mondo della produzione. Essi sono talmente importanti per vendere i prodotti al punto che per la iGuzzini illuminazione il vero cliente è il prescrittore e non l'utilizzatore o committente del progetto. L'agente monomandatario è gestore della relazione con il prescrittore la cui immagine, in alcuni casi come quello dell'architetto Renzo Piano, è talmente forte da offuscare sia il distributore che l'impresa produttrice. Nel tradizionale rapporto fra impresa produttrice e distributore s'inserisce il prescrittore che ridefinisce persino il ruolo del distributore; questo mantiene il controllo della funzione logistica, economico-finanziaria e comunicativa ma deve necessariamente interfacciarsi con $i$ prescrittori per rifornire il committente, ridimensionando le sue funzioni informative. L'installatore infine viene chiamato in causa solo nella fase d'installazione ma ben poca importanza riveste nella fase pre-acquisto. Fra gli attori del canale quindi si innescano rapporti interattivi e di reciproca interdipendenza che, come sostengono Norman e Ramirez (1993), determinano il superamento del tradizionale concetto di catena del valore in favore di una costellazione del valore. Seguendo questo approccio, l'impresa crea valore coinvolgendo tutti gli attori che possono arricchire il valore dell'offerta e gestendo efficientemente le relazioni che intercorrono fra gli attori coinvolti.

Il mercato del lighting si presenta con una struttura complessa in cui le imprese manifatturiere per creare valore devono adottare un approccio olistico per coinvolgere e gestire in una costellazione del valore tutti quegli attori che possono contribuire all'arricchimento dell'offerta.

I dati raccolti indicano che all'interno dell'impresa indagata vi sia una forte consapevolezza dell'importanza del servizio, nonostante il fatturato dipenda dal numero dei prodotti materiali venduti e l'azienda venga classificata secondo gli schemi tradizionali come un'impresa manifatturiera. Gli intervistati riconoscono che i servizi trainano le vendite degli impianti di illuminazione, determinando il 
posizionamento nella parte alta del mercato del lighting, impattando favorevolmente sull'immagine dell'azienda, che secondo gli intervistati dipende fortemente dalla qualità dei servizi erogati (4.1). I risultati dello studio mettono in evidenza come la cultura organizzativa interna service-oriented sia un elemento chiave nell'implementazione della servitization (Martinez et al., 2010) ed indicano altresì che la cultura gioca un ruolo cruciale per definire la qualità del servizio ed il posizionamento dell'impresa. Perciò:

La consapevolezza dell'importanza del servizio è condizione necessaria ma non sufficiente per un'efficace implementazione di un'offerta integrata e determinante per la qualità del servizio ed il posizionamento dell'impresa.

Nel sistema d'offerta della iGuzzini illuminazione il bene fisico viene combinato con una serie di servizi per qualificare il pacchetto di base (4.2). Il servizio principale è la progettazione illuminotecnica, che in una prima fase del ciclo di vita del settore poteva essere visto come un servizio di differenziazione. Attualmente, invece, la progettazione viene considerata come un servizio necessario nella relazione con il prescrittore e gli altri servizi possono essere qualificati come servizi di facilitazione. Il caso conferma quindi le riflessioni di Grönroos (2009) il quale sostiene che la distinzione fra servizi di facilitazione e servizi di differenziazione può cambiare a seconda del contesto o del periodo considerato. Infatti un servizio che nelle fasi iniziali dello sviluppo del settore può essere di facilitazione, può diventare necessario nella fase di maturità oppure uno stesso servizio può essere di facilitazione pr un segmento e necessario in un altro. Inoltre, a seconda della tipologia d'offerta, esisteranno differenti opportunità di arricchire il pacchetto di base con i servizi (Martinez et al., 2010). Vengono inoltre confermate le riflessioni di Kotler (1967) sull'importanza di considerare fra gli interlocutori dell'impresa anche il prescrittore nel processo di creazione del valore, e di Ordanini (1999) sulla necessità dell'impresa di arricchire le competenze alla base del vantaggio competitivo dell'impresa cliente. Aspetto innovativo che emerge dal caso, emblematico di imprese produttrici di beni complessi ad alto contenuto di conoscenza, simbolismo ed immaterialità, è l'importante ruolo svolto dagli strumenti tecnologici per la creazione di applicazioni multimediali e dalle risorse umane dedicate al loro utilizzo. La progettazione illuminotecnica si basa infatti sulla conoscenza di specifici software per svolgere calcoli complicati (Dialux, Rialux) e software per la realizzazione di applicazioni multimediali (Photoshop, 3DMax studio). La combinazione di strumenti e risorse umane dedicate consente quindi di creare nuovi servizi e migliorare l'efficacia della comunicazione dell'intero pacchetto d'offerta. In sintesi:

La dotazione di strumenti per lo sviluppo di applicazioni multimediali e la dotazione di risorse umane dedicate, offrono molte opportunità per creare nuovi servizi destinati ai vari interlocutori che entrano in gioco nel processo di vendita e 
consentono di migliorare l'efficacia della comunicazione dell'intero pacchetto d'offerta.

La partecipazione, l'accessibilità e l'interazione con il cliente, fondamentali nell'erogazione del servizio nel modello di Grönroos, sono considerate importanti dimensioni anche per l'implementazione della servitization. La complessità della struttura del mercato richiede tuttavia di rivedere il modello di Grönroos ampliando il numero di soggetti da considerare nella creazione di valore. Il modello infatti non può considerare solamente il rapporto fra imprese e cliente ma deve includere anche tutti gli attori che in vario modo contribuiscono ad aumentare il valore dell'offerta. La partecipazione, l'accessibilità e l'interazione quindi dovranno essere definite tenendo ben presente il contributo ed il ruolo dei soggetti in gioco.

Inoltre, dalle evidenze emergono ulteriori interessanti indicazioni sulle sottodimensioni del modello. Innanzitutto, la mancanza di simultaneità fra l'erogazione e la produzione del servizio fa perdere di significato all'accessibilità temporale e fisica. Anche l'interazione con gli altri clienti viene ridimensionata in quanto sono difficilmente immaginabili situazioni simili a quelle dei clienti in coda allo sportello bancario. Dall'altra parte è necessario considerare che l'agente assume un peso rilevante nella gestione delle relazioni fra impresa, distributore e prescrittore. Nella iGuzzini illuminazione infatti l'agente, in linea con la letteratura sul tema (Guenzi, 2002; Pencarelli e Cioppi, 2008), svolge un ruolo strategico poiché è un "vigile" della relazione stimolando e facilitando in tutte le fasi del progetto la partecipazione e la condivisione della soluzione fra più interlocutori. Inoltre, l'accessibilità del cliente business può essere espressa principalmente come accessibilità informativa in quanto il prescrittore ha essenzialmente bisogno di informazioni e dati tecnici e di essere continuamente supportato nella scelta di una soluzione illuminotecnica. Di conseguenza, ci sarà un'attenzione crescente alla tecnologia, come ad esempio il catalogo on-line, per soddisfare il fabbisogno informativo del prescrittore che rappresenta un sistema che agevola l'accessibilità informativa e comunicativa e facilita le interazioni tra impresa e vari attori del network del valore. Da non sottovalutare infine che l'eterogeneità delle situazioni che devono essere affrontate rende estremamente difficile la pianificazione formale dell'accessibilità, dell'interazione e della partecipazione.

L'accessibilità, la partecipazione e l'interazione risultano essere dimensioni importanti per l'implementazione della servitization e devono essere definite tenendo presente tutti i soggetti coinvolti nella costellazione del valore. Queste sono difficilmente pianificabili, ma possono essere gestite sfruttando le applicazioni tecnologiche e conferendo all'agente di vendita un'adeguata autorità $e$ responsabilità in una relazione fra più interlocutori.

Dai dati raccolti emerge che la partecipazione del prescrittore crea valore in particolar modo per la capacità di arricchire il patrimonio di conoscenze dell'impresa mentre i ritorni economici di breve periodo sono posti in secondo 
piano. È facile desumere inoltre che la iGuzzini illuminazione, attraverso il prescrittore, acceda anche ad un insieme di relazioni sviluppate dallo stesso prescrittore e sfrutti l'immagine di questo creatasi nel tempo. Le relazioni di lungo periodo sono favorite dall'interdipendenza del settore del lighting con il settore edile e proprio da questa interdipendenza derivano rapporti su più anni in continua evoluzione. Sarà fondamentale per l'impresa quindi garantire la stabilità dei rapporti di lavoro con i propri dipendenti in modo tale che si possano avere con i prescrittori delle continue relazioni di scambio informativo e possano innescarsi dei meccanismi di reciproco apprendimento.

La relazione con il prescrittore richiede l'impiego di risorse umane con contratti stabili al fine di sfruttare al massimo le competenze, le esperienze, il network di relazioni e l'immagine del prescrittore.

Dalle interviste si evince che la comunicazione al prescrittore necessita soprattutto di informazioni sugli aspetti tecnici ed il confine fra formazione e comunicazione è labile. La maggiore difficoltà non è data dalla comunicazione dell'intangibilità del servizio, ma dal comunicare l'importanza e la necessità dei servizi iGuzzini illuminazione. Non a caso negli anni ' 80 l'impresa ha sfruttato i mass-media per instillare ad un pubblico ampio l'idea dell'importanza della progettazione illuminotecnica e solo la consapevolezza sul valore della progettazione ha permesso di sviluppare relazioni e, successivamente, di acquisire progetti. Per mantenere queste relazioni è indispensabile una formazione continua del personale il quale deve essere preparato a rispondere ad ogni istanza dei prescrittori e proprio per questo può essere considerato un part-time marketer (Gummesson, 2006) in quanto interagisce con i prescrittori per definire un adeguato progetto illuminotecnico. La difficoltà nel comunicare l'importanza dello studio illuminotecnico è evidente per i prescrittori dei Paesi in via di sviluppo come la Cina, l'India ed il Brasile, i quali non avendo ancora sviluppato una sensibilità verso la progettazione illuminotecnica sono fortemente influenzati dal prezzo nelle loro scelte di acquisto. Si deduce quindi che l'implementazione della servitization non possa trascurare azioni finalizzate a creare una domanda formata ed informata sui benefici e sull'importanza dei servizi. Gli sforzi nella ridefinizione dei processi interni quindi potrebbero essere vanificati se il mercato finale ed intermedio non fosse preparato a recepire i benefici dei servizi sviluppati.

Per un'efficace implementazione della servitization sono necessarie continue azioni di formazione al personale interno dell'impresa ma anche al mercato per accrescere la consapevolezza della domanda sul valore dei servizi, allineandone le attese con le potenzialità dell'offerta. 
Fig. 2: Modello dell'offerta incrementata risultante dal caso

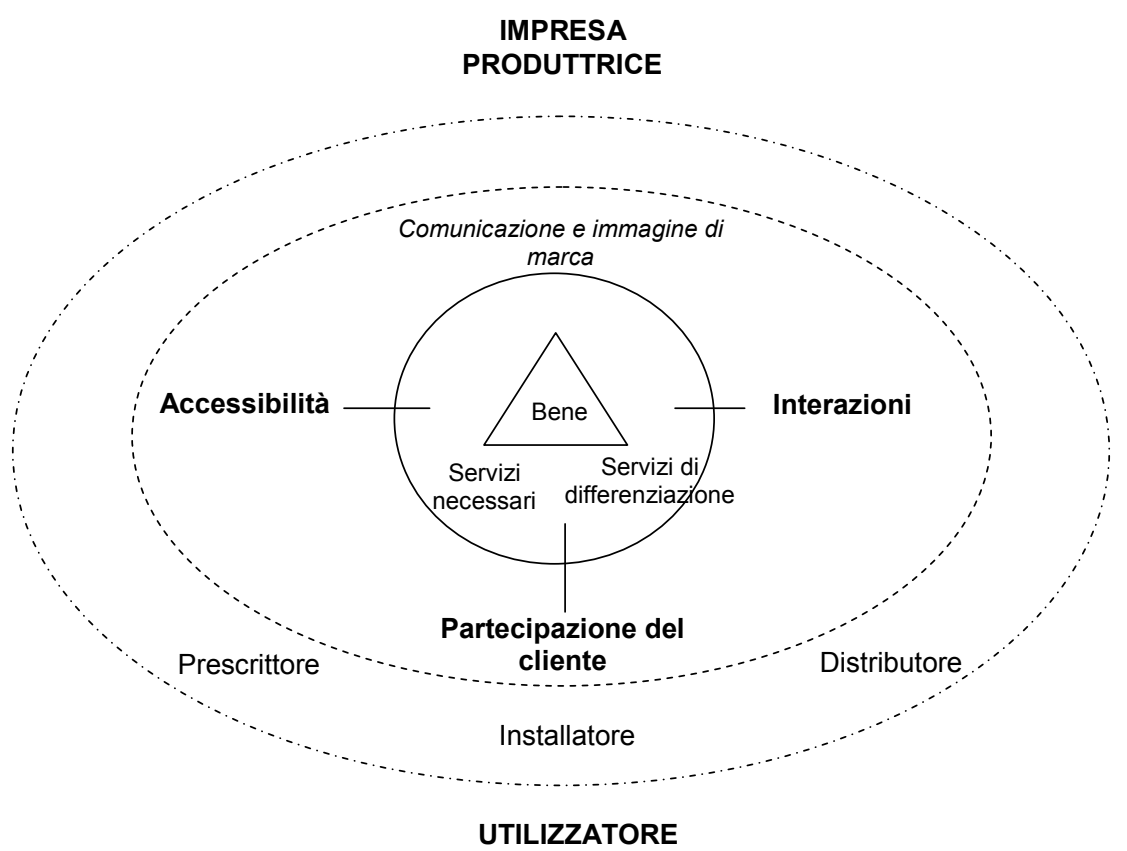

Fonte: Adattamento da Grönroos, 2009

\section{Implicazioni, conclusioni e limitazioni}

Il caso fornisce interessanti implicazioni manageriali per le imprese manifatturiere che intendono riqualificare la propria offerta di valore aggiungendo componenti di servizio, di conoscenza e di immaterialità al portafoglio prodotti. Dallo studio emerge in particolare che le imprese che puntano sulla servitization devono possedere e comunque far leva su una profonda cultura del servizio: quindi, nell'attuare i processi di service management, devono assicurarsi di diffondere e condividere la cultura del servizio tra gli attori interni ed esterni all'impresa coinvolti nella co-creazione di valore, secondo il paradigma del Service Dominant Logic. L'orientamento culturale al servizio, fra l'altro, garantisce quella flessibilità nell'erogazione dell'offerta necessaria per gestire situazioni poco pianificabili e relazioni sempre più personalizzate. Risulta inoltre che ai fini di una servitization capace di coinvolgere la pluralità di attori coinvolti nella costellazione del valore, l'impresa deve saper sfruttare al meglio le opportunità offerte dalla tecnologia, sfruttando ad esempio piattaforme aperte e condivise entro cui far circolare informazioni e conoscenze utili alla co-generazione di valore in una prospettiva di open innovation (Di Maria e Bettiol, 2009); oltre alle risorse tecnologiche, non 
vanno trascurate naturalmente le risorse umane qualificate, di cui un'azienda che punta ad aumentare il valore mediante processi di servitization deve dotarsi anche al fine di innovare continuamente l'offerta in collaborazione con i vari interlocutori di cui occorre saper decodificare e comporre linguaggi assai differenti, come nel caso di potenziali acquirenti non specializzati o di prescrittori professionisti ed avanguardie nel settore. Di qui, la crucialità e l'importanza di azioni di formazione continua rivolta al personale interno oltre che al mercato, al fine di rendere maggiormente consapevole la domanda e gli attori della filiera in merito alle potenzialità di valore contenute nell'offerta aziendale.

Va segnalato inoltre come dallo studio emerga che il modello di Grönroos, arricchito con le indicazioni sopra richiamate (Fig. 2), possa essere considerato un valido strumento concettuale per i manager di qualsiasi tipologia d'impresa che necessitino di riprogettare ed innovare il sistema d'offerta per rafforzare il posizionamento competitivo in un mondo di "concorrenza di servizi", creando i presupposti per introdurre efficaci sistemi di misurazione della qualità percepita di offerte ad alto contenuto di servizio (Baccarani et al., 2010). In particolare, grazie al modello concettuale proposto, si riesce a mostrare come un'impresa operante in un comparto di beni problematici come quello del lighting riesca ad aumentare il valore dell'offerta attuando processi di servitization mediante il coinvolgimento di network di attori interni ed esterni all'impresa: il personale, i prescrittori, la rete di vendita e gli stessi acquirenti.

Il principale limite del lavoro è che considera un solo caso, che per quanto rappresentativo di come procedere verso la servitization all'interno di un settore di beni problematici ad alta complessità e contenuto immateriale, come il lighiting, non consente eccessive generalizzazioni. Inoltre, lo studio non approfondisce il legame tra servitization e le performance e non vengono analizzati in dettaglio i ritorni sia economici che d'immagine che derivano da una strategia di questo tipo.

Sono auspicabili quindi nuove ricerche in altri settori per comprendere ulteriori modalità d'implementazione di strategie di servitization e studi quantitativi per testare le relazioni fra le variabili individuate. Infine, un'ulteriore linea di ricerca futura può essere la verifica della sostenibilità economica dell'erogazione gratuita dei servizi nel lungo periodo.

\section{Bibliografia}

AHAMED Z., KAMOSHIDA A., INOHARA T. (2013), "Organizational Factors to the Effectiveness of Implementing Servitization Strategy”, Journal of Service Science and Management, n. 6, pp. 177-185.

BACCARANI C., UGOLINI M., BONFANTI A. (2010), "A conceptual service quality map: The value of a wide opened perspective", Atti del Convegno Organizational Excellence in Service, University of Coimbra - Portugal, 2-4 September.

BAINES T.S., LIGHTFOOT H.W., BENEDETTINI O., KAY J.M. (2009), "The servitization of manufacturing: A review of literature and reflection on future challenges", Journal of Manufacturing Technology Management, vol. 20, n. 5, pp. 547-567. 
COOK M.B., BHAMRA T.A., LEMON M. (2006), "The transfer and application of Product Service Systems: from academia to UK manufacturing firms", Journal of Cleaner Production, vol. 14, n. 17, pp. 1455-1465.

CORBETTA P. (1999), Metodologia e tecniche della ricerca sociale, Il Mulino, Bologna.

COYNE K. (1989), "Beyond service fads - Meaningful strategies for the real world", Sloan Management Review, vol. 30, n. 4, pp. 69-76.

DI MARIA E., BETTIOL M., (2009), "Il management dell'innovazione", in Bernardo B., Gandolfi V., Tunisini A., (a cura di), Economia \& Management delle imprese, Hoepli, Milano.

EISENHARDT K.M. (1989), "Making fast strategic decisions in high-velocity environments" Academy of Management Journal, vol. 32, n. 3, pp. 543-576.

FATTORE G. (2005), Metodi di ricerca in economia aziendale, Egea, Milano.

GEBAUER H., FRIEDLI T. (2005), "Behavioral implications of the transition process from products to services", Journal of Business and Industrial Marketing, vol. 20, n. 2, pp. 70-78.

GRÖNROOS C. (2009), Management e marketing dei servizi. La gestione del cliente nel mercato dei servizi, Isedi, Torino.

GOEDKOOP M.J., VAN HALEN C.J.G., TE RIELE H.R.M., ROMMENS P.J.M. (1999), Product Service Systems, Ecological and Economic Basics. Report for Dutch Ministries of Environment (VROM) and Economic Affairs (EZ).

GUENZI P. (2002), La vendita relazionale. La gestione dei processi commerciali nella prospettiva del Relationship Selling, Etas, Milano.

GUMMESSON E. (2006), Marketing relazionale: gestione del marketing nei network di relazioni, Hoepli, Milano.

JICK T.D. (1979), "Mixing Qualitative and Quantitative Methods: Triangulation in Action", Administrative Science Quarterly, vol. 24, n. 4, pp. 602-611.

KOTLER P. (1967), Marketing Management: Analysis, Planning and Control, Prentice Hall, New York.

LAMBERTI L., NOCI G. (2010), "Marketing strategy and marketing performance measurement system: Exploring the relationship", European Management Journal, vol. 28 , n. 2, pp. $139-152$.

LEVITT T. (1972), "Production-line approach to service", Harvard Business Review, September-October, pp. 41-52.

LOJACONO G. (2005), Trend evolutivi e modifica dei business model nel settore dell'illuminazione, FrancoAngeli, Milano.

MARTINEZ V., BASTL M., KINGSTON J., EVANS S. (2010), "Challenges in transforming manufacturing organisations into product-service providers", Journal of Manufacturing Technology Management, vol. 21, n. 4, pp. 449-469.

MATHE H., SHAPIRO R.D. (1993), Integrating Service Strategy in the Manufacturing Company, Chapman and Hall, London

MATHIEU V. (2001), "Service strategies within the manufacturing sector: Benefits, costs and partnership", International Journal of Service Industry Management, vol. 12, n. 5, pp. 451-475.

MANZINI E., VEZOLLI C. (2003), “A strategic design approach to develop sustainable product service systems: examples taken from the "environmentally friendly innovation' Italian prize”, Journal of Cleaner Cleaner Production, vol. 11, pp. 851857. 
MENON A., HOMBURG C., BEUTIN N. (2005), "Understanding Customer Value in Business-to-Business Relationships", Journal of Business-to-Business Marketing, vol. 12, n. 2, pp. 1-38.

NEELY A. (2009), "Exploring the financial consequences of the servitization of manufacturing", Operations Management Research, vol. 1, n. 2, pp. 103-118.

NORMANN R., RAMIREZ R. (1995), Le strategie interattive d'impresa: dalla catena alla costellazione del valore, Etas libri, Milano.

OLIVA R., KALLENBERG R. (2003), "Managing the transition from products to services", International Journal of Service Industry Management, vol. 14, n. 2, pp. 160-172.

ORDANINI A. (1999), Servizi alle imprese e vantaggio competitivo: informazione, conoscenza e aspetti relazionali nella gestione di impresa, EGEA, Milano.

PAIOLA M., RULLANI E. (2009), "Servizi e competitività nell'economia dell'immateriale", in Bernardo B., Gandolfi V., Tunisini A., (a cura di), Economia \& Management delle imprese, Hoepli, Milano.

PASTORE A., VERNUCCIO M. (2006), Impresa e comunicazione. Principi e strumenti per il management, Apogeo, Milano.

PATTON M. Q. (2002), Qualitative Research \& Evaluation Methods, SAGE Publications, Beverly Hills.

PENCARELLI T. (2013), "Processi di service management", in Baglieri D., Dagnino G. B., Faraci R., (a cura di), Management dell'impresa, ISEDI, Torino.

PENCARELLI T., CIOPPI M. (2008), "I processi di vendita ed il marketing delle PMI", in Tunisini A., (a cura di), Teorie e applicazioni di business marketing. Comprendere $i$ processi di mercato e modellare l'azione di management, Franco Angeli Milano.

PRAHALAD C.K., RAMASWAMY V. (2000), "Co-opting customer competence", Harvard Business Review, vol. 78, n. 1, pp. 79-87.

RISPOLI M., TAMMA M. (1992), "Beni e servizi, cioè prodotti”, Sinergie, n. 29, pp. 95-113.

TUNISINI A. (2002), "Riflessioni sulla gestione del prodotto nei mercati industriali", Micro \& Macro Marketing, vol. XI, n. 2, pp. 217-232.

ULAGA W., CHACOUR S. (2001), "Measuring Customer-Perceived Value in Business Markets: A Prerequisite for Marketing Strategy Development and Implementation", Industrial Marketing Management, vol. 30, n. 6, pp. 525-540.

VANDERMERWE S., RADA J. (1988), "Servitization of business: Adding value by adding services", European Management Journal, vol. 6, n. 4, pp. 314-324.

VANDERMERWE S., RADA J. (1989), "European manufacturers shape up for services", The Journal of Business Strategy, vol. 10, n. 6, pp. 42-46.

VARGO S.L., LUSCH R.F. (2004), "Evolving to a New Dominant Logic for Marketing", Journal of Marketing, vol. 68, n. 1, pp. 1-17.

VARGO S.L., LUSCH R.F. (2007), "Service-dominant logic: continuing the evolution", Journal of the Academy of Marketing Science, vol. 36, n. 1, pp. 1-10.

WISE R., BAUMGARTNER P. (1999), "Go downstream: The new profit imperative in manufacturing", Harvard Business Review, vol. 77, n. 5, pp. 133-141.

YIN R.K. (2009), Case Study Research: Design and Methods, SAGE Publications, Beverly Hills.

ZEITHAML V.A. (1988), "Consumer perceptions of price, quality, and value: A means-end model and synthesis of evidence", Journal of Marketing, vol. 52, n. 3, JULY, pp. 2 22. 
